\title{
Functional characterization of osteosarcoma cell lines provides representative models to study the human disease
}

\author{
Alexander B Mohseny ${ }^{1}$, Isidro Machado², Yongping Cai ${ }^{1,3}$, Karl-Ludwig Schaefer ${ }^{4}$, Massimo Serra ${ }^{5}$, \\ Pancras CW Hogendoorn ${ }^{1}$, Antonio Llombart-Bosch ${ }^{2}$ and Anne-Marie Cleton-Jansen ${ }^{1}$
}

Cancer cell lines represent in vitro models for studying malignancies, general cell biology, drug discovery and more. Whether they can be considered as exact representative models of the parental tumors remains uncertain given the acquisition of additional ex vivo changes of the cells and the lack of tissue architecture and stroma. Previously, within the EuroBoNeT consortium, we characterized a collection of bone sarcoma cell lines on genomic and proteomic level. Here, we address the phenotypical and functional characterization of the unique set of osteosarcoma cell lines $(n=19)$ in vitro and in vivo. For functional analysis of differentiation capacity, cells were stimulated towards osteoblasts, adipocytes and chondrocytes. Furthermore, all cell lines were injected subcutaneously and intramuscularly into nude mice to assay their in vivo tumor formation capacity as well as for phenotypical analysis of the tumors. All formed tumors were further characterized histologically and immunohistochemically. Out of 19 cell lines, 17 (89\%) showed adipogenic differentiation, $13 / 19(68 \%)$ could differentiate towards osteoblasts and in 6/19 (32\%) cell lines chondrogenic differentiation was evident. About half of the cell lines $(8 / 19,42 \%)$ produced tumors in vivo after subcutaneous and intramuscular injections. Several cell lines showed invasion into adjacent tissues and one tumor developed several lung metastases. The use of cell lines, especially in cancer research, is of paramount importance. Here, we identify comprehensively characterized osteosarcoma cell lines, which robustly represent clinical osteosarcoma providing researchers useful in vitro and in vivo models to study the genetics and functional characteristics of this highly malignant neoplasm.

Laboratory Investigation (2011) 91, 1195-1205; doi:10.1038/labinvest.2011.72; published online 25 April 2011

KEYWORDS: contamination; HOS; misidentification; MNNG; origin; tumorigenesis; U2OS

Tumor cell lines have been considered as instrumental entries into tumor cell biology and often used for studying the mechanisms of carcinogenesis, the functional characteristics of genes and drug discovery, screening and response. ${ }^{1-8}$ Nevertheless, concerns have been raised about the use of cell lines that can be divided into two categories of criticism. First, mainly due to poor experimental conduct, false (derived from another cell population within the cell culture than the one intended), cross-contaminated (with other human cells or cells from other species) and/or pathogen- (mycoplasm) contaminated cell lines have been discovered repeatedly. ${ }^{9-12}$ Second, the representativeness of cultured cell lines as compared with the original tumors is being questioned as these cells have been cultured in the absence of stroma, ${ }^{13}$ hence lacking the proper microenvironment and the original tissue architecture. Moreover during culturing, specific cells are continuously selected based on the in vitro conditions and cells might undergo additional ex vivo mutations. On the other hand, it has been shown that cell lines adequately represent the tumors they are originating from, especially at the genetic level. ${ }^{14-19}$ All in all cell lines appear to be adequate models as long as there are controlled culturing conditions and a good selection process to identify the appropriate ones. ${ }^{10,11,20}$ Accordingly, to select osteosarcoma cell lines representative of human osteosarcoma, here we characterize 19 cell lines in vitro and in vivo by using robust methods subjected to regular quality control.

\footnotetext{
${ }^{1}$ Department of Pathology, Leiden University Medical Center, Leiden, The Netherlands; ${ }^{2}$ Department of Pathology, University of Valencia, Valencia, Spain; ${ }^{3}$ Department of Pathology, School of Medicine, Shandong University, Jinan, China; ${ }^{4}$ Institute of Pathology, University Medical Center Duesseldorf, Duesseldorf, Germany and

${ }^{5}$ Laboratorio di Oncologia Sperimentale, Istituto Ortopedico Rizzoli, Bologna, Italy

Correspondence: Dr A-M Cleton-Jansen, Department of Pathology, Leiden University Medical Center, PO Box 9600, L1-Q, Leiden 2300 RC, The Netherlands.

E-mail: a.m.cleton-jansen@lumc.nl

Received 29 October 2010; revised 24 February 2011; accepted 6 March 2011
} 
Osteosarcoma is a highly malignant tumor, fatal for about one-third of the patients who do not respond to chemotherapy and alternative therapies are still missing. This is mainly due to the rarity and the high genetic heterogeneity of these tumors, which makes it difficult to have patient cohorts that are large enough to compensate for the high genetic variability. One way to bypass this problem is to study osteosarcoma-derived cell lines, which are abundantly used as in vitro models because they are highly proliferative and receptive for genetic manipulation by transfection. Recently, through the effort of the EuroBoNet network, a large panel of osteosarcoma cell lines, among other bone sarcoma cell lines, was described genetically. ${ }^{18}$ To select the cell lines that are most representative of primary osteosarcoma, here we analyzed their in vitro differentiation capacity and their in vivo tumorigenicity in nude mice. Furthermore, generated tumors were histologically and immunohistochemically classified.

By this comprehensive study, we were able to identify at least eight cell lines, which convincingly represent primary human osteosarcoma. HOS-143B was discovered to be highly metastatic to the lungs of the animals after subcutaneous and intramuscular xenotransplantations. The cell lines characterized here represent excellent in vitro and especially in vivo models to obtain a better understanding of osteosarcoma biology, and subsequently the identification of novel targets for specific therapies.

\section{MATERIALS AND METHODS Cell Lines}

Cells were provided by the different partner institutes of the EuroBoNet network ${ }^{21-25}$ or derived from ATCC and were grown in RPMI 1640 (Invitrogen, Karlsruhe, Germany) supplemented with 2\% L-glutamine (Invitrogen) and 10\% fetal calf serum under standard conditions. Control tests for mycoplasm contamination were carried out routinely (two times a month), using a PCR-based commercially available detection kit according to the manufacturer's protocol (VenorGem, Minerva Biolabs, Berlin, Germany). Furthermore, all cell lines and xenografts were genotyped using the Powerplex 1.2 system (Promega, Leiden, the Netherlands), as described previously, ${ }^{26}$ to match the cells to their previously published identities and to detect any cross-contaminations. ${ }^{18}$ Table 1 summarizes the characteristics and the culturing conditions of the cell lines.

\section{In Vitro Differentiation}

Before differentiation, alkaline phosphatase (ALP) activity was measured for all cell lines to determine their basal ALP activity. Differentiation studies were performed as described previously: ${ }^{27}$ cells were induced to differentiate into mature mineralizing osteoblasts over a 3-week culture period by plating $10^{4}$ cells in $500 \mu \mathrm{l}$ medium per well of a 24 -well plate (for ALP measurement) and by plating $2 \times 10^{4}$ cells in $1 \mathrm{ml}$ medium per well of a 12 -well plate (for mineralization). Ascorbic acid $(50 \mu \mathrm{g} / \mathrm{ml})$ was added to the medium starting
Table 1 Origin and characteristics of the cell lines

\begin{tabular}{|c|c|c|c|c|c|}
\hline Cell line & PD $(h)^{a}$ & Subtype $^{\mathrm{b}}$ & $\mathrm{Age}^{\mathrm{c}}$ & Sex ${ }^{c}$ & Reference $^{d}$ \\
\hline HOS & 36 & N/A & 13 & $\mathrm{~F}$ & ATCC-CRL-1543 \\
\hline HOS-143B & 36 & $\mathrm{~N} / \mathrm{A}$ & 13 & $\mathrm{~F}$ & ATCC-CRL-8303 \\
\hline HOS-MNNG & 24 & N/A & 13 & $\mathrm{~F}$ & ATCC-CRL-1547 \\
\hline OSA & 24 & Fibroblastic & 19 & M & ATCC-CRL-2098 \\
\hline MG-63 & 48 & Fibroblastic & 14 & M & ATCC-CRL-1427 \\
\hline Saos-2 & 48 & N/A & 11 & $\mathrm{~F}$ & ATCC-HTB-85 \\
\hline U2OS & 36 & $\mathrm{~N} / \mathrm{A}$ & 15 & $\mathrm{~F}$ & ATCC-HTB-96 \\
\hline IOR/MOS & 72 & Osteoblastic & 13 & $\mathrm{~F}$ & Benini et $a l^{21}$ \\
\hline IOR/OS9 & 72 & Osteoblastic & 15 & M & Benini et $a l^{21}$ \\
\hline IOR/OS10 & 72 & Fibroblastic & 10 & $\mathrm{~F}$ & Benini et $a l^{21}$ \\
\hline IOR/OS14 & 48 & Osteoblastic & 13 & M & Benini et $a l^{21}$ \\
\hline IOR/OS15 & 48 & Osteoblastic & 12 & $\mathrm{~F}$ & Benini et $a l^{21}$ \\
\hline IOR/OS18 & 60 & Osteoblastic & 33 & M & Benini et $a l^{21}$ \\
\hline SARG & 72 & N/A & 25 & M & Benini et $a l^{21}$ \\
\hline KPD & 36 & Osteoblastic & 7 & $\mathrm{~F}$ & Bruland et $a l^{22}$ \\
\hline $\mathrm{OHS}$ & 36 & Osteoblastic & 14 & M & Fodstad et $a^{23}$ \\
\hline HAL & 48 & N/A & 16 & M & Høifødt, Oslo ${ }^{e}$ \\
\hline ZK-58 & 72 & Osteoblastic & 21 & M & Schulz et $a l^{24}$ \\
\hline MHM & 60 & Fibroblastic & 41 & $\mathrm{~F}$ & Kjonniksen et $a^{25}$ \\
\hline
\end{tabular}

PD, population doubling; NA, not available.

For each cell line used in this study, the in vitro growth speed, the subtype of the originating tumor, the age and the gender of the donor and a reference for the cell line are depicted, respectively.

${ }^{a}$ Average time needed for one PD in hours.

b Osteosarcoma subtype of the original tumor from which cells were derived, note that this information is not available for all cell lines, indicated as N/A.

${ }^{\mathrm{C}}$ Age and the sex of the patient from whom the tumor originates.

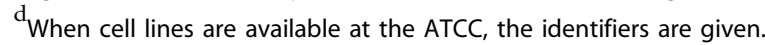

Enpublished.

on day 4 and $\beta$-glycerolphosphate $(5 \mathrm{mM})$ on day 11 . Osteoblastic differentiation was quantified as described previously, ${ }^{28}$ with some modification. In short, cells were lysed in ALP lysis buffer $\left(10 \mathrm{mmol} / \mathrm{l}\right.$ glycine, $0.1 \mathrm{mmol} / 1 \mathrm{MgCl}_{2}$, $10 \mu \mathrm{mol} / 1 \mathrm{ZnCl}_{2}, 0.1 \%$ Triton $\left.\mathrm{X}-100\right)$ and $25 \mu \mathrm{l}$ was used to determine ALP activity using $6 \mathrm{mmol} / \mathrm{l} p$-nitrophenylphosphate as a substrate and measuring absorbance at $405 \mathrm{~nm}$ on an ELISA reader. For comparisons, ALP activity was calculated per $\mu$ l per minute until saturation. The presence of mineralization was assessed by staining with Alizarin Red S $(20 \mathrm{mg} / \mathrm{ml}, \mathrm{pH}$ 5.5). Adipogenic differentiation was induced by culturing $10^{4}$ cells in $500 \mu \mathrm{l}$ medium per well of a 24-well plate for 2 weeks. FBS was replaced by $10 \%$ charcoal-stripped FBS in the basal medium and indomethacin $(50 \mu \mathrm{M})$ was added starting on day 4 . Adipocytes containing lipid droplets were stained with Oil Red $\mathrm{O}(3 \mathrm{mg} / \mathrm{ml})$. For chondrogenic differentiation, cells were cultured as pellets $\left(2 \times 10^{5}\right.$ cells per 
pellet) in U-shaped 96-well plates containing $200 \mu \mathrm{l}$ DMEM supplemented with $\mathrm{P} / \mathrm{S}(1 \%)$, pyruvate $(100 \mu \mathrm{g} / \mathrm{ml})$, transferrin selenite $(10 \mu \mathrm{l} / \mathrm{ml})$ and proline $(40 \mu \mathrm{g} / \mathrm{ml})$. During the first 2 weeks, ascorbic acid $(50 \mu \mathrm{g} / \mathrm{ml})$, TGF- $\beta 3(10 \mathrm{ng} / \mathrm{ml})$ and dexamethasone $\left(10^{-7} \mathrm{M}\right)$ were added to the medium. Starting at the third week, ascorbic acid $(50 \mu \mathrm{g} / \mathrm{ml})$, BMP6 $(500 \mathrm{ng} / \mathrm{ml})$ and $\beta$-glycerolphosphate $(5 \mathrm{nM})$ were added. After 5 weeks, pellets were fixed in formalin and embedded in paraffin and sections were stained with toluidine blue to identify chondrogenic matrix.

\section{In Vivo Tumor Formation}

After trypsinization, cells were counted and dilutions of $2 \times 10^{6}$ cells in $10 \mu \mathrm{l}$ phosphate-buffered saline were prepared. Each cell line was injected into one nude mouse at three locations: two subcutaneous injections on the back (upper left and lower right corner) and one intramuscular injection (upper part of the hind left paw). After the injections, tumor growth was screened twice a week by observation and palpation. Animals were killed when tumors reached approximately $1 \mathrm{~cm}$ in diameter or no sign of tumor formation was detected after 6 months or when any kind of animal suffering was detected. All cell lines that did not produce tumors were re-injected into a second mouse (again at three locations) to validate this observation. After being killed, all tumors were surgically removed and archived by freezing as well as by fixing in formalin and embedding in paraffin. Furthermore, a complete autopsy was performed to detect invasion, angiogenesis and/or metastases of the tumors. Growth into adjacent tissues was labeled as 'invasion' only when after subcutaneous injection cells invaded underlying tissues (mostly muscle) and only when this was confirmed not to be caused by direct injection into those tissues. (Neo)-angiogenesis was considered when blood vessels were identified inside the tumor's mass at locations where they were not anatomically expected and/or when vessel subcutaneously grew towards the tumors. All animal experiments were performed according to the Dutch and Spanish animal experiments guidelines, and approved by the Valencia University Animal Experiments Committee.

\section{Tissue Array Construction}

Tissue cores from formalin-fixed and paraffin-embedded (FFPE) tumor areas selected by two pathologists (IM and ALLB) on the basis of a hematoxylin and eosin (H\&E)stained slide were taken from each specimen (Beecher Instruments, Silver Springs, MD, USA). The cores $(2 \mathrm{~mm}$ diameter) were arrayed on a recipient paraffin block using a tissue arrayer from Beecher Instruments. ${ }^{29}$ At least three cores from each tumor were sampled to outweigh intratumoral heterogeneity. ${ }^{30}$ Each tissue array contains additional cores from other tissue types both as internal controls for immunohistochemistry (IHC) as well as for orientation purposes.

\section{Staining}

IHC was performed on FFPE tissue array sections as described previously. ${ }^{31}$ Next to the H\&E, Periodic acid-Schiff and Masson's trichrome stainings to assay the histology of the xenografts, the slides were stained with all antibodies listed in Table 3, which also summarizes the IHC conditions. All stainings in this study were independently evaluated by two pathologists (IM and ALLB) as previously described in detail. $^{32,33}$ Intensity and percentage of positive neoplastic cells were evaluated. Cellular localization of immunopositivity (nuclear, cytoplasmic or membranous) was also recorded. Conflicting assessments were reviewed until final agreement was achieved. A final immunopositivity score was indicated per sample as negative $(-)$, weakly positive $(+)$, moderately positive $(++)$ or strongly positive $(+++)$.

\section{HOS-143B}

To study the progression of the only metastatic cell line, HOS-143B, cells were injected two more rounds in 5 and 10 animals, respectively. In addition from one of the primary tumors, a piece was subcutaneously transplanted to another recipient nude mouse, and this was repeated three times. Both from subcutaneous and intramuscular tumors, lung metastases were analyzed for p53 protein expression and mutation state as described previously. ${ }^{18,34,35}$

\section{RESULTS \\ Differentiation Capacity of Osteosarcoma Cell Lines}

Differentiation towards at least one of the three lineages was detected in all cell lines, 14 out of 19 cell lines could differentiate towards at least two lineages and three cell lines (OSA, IOR/OS9 and IOR/OS18) differentiated into all three lineages (Table 2). In 17/19 (89\%) cell lines, adipogenic differentiation was observed, 13/19 (68\%) could differentiate towards osteoblasts and in 6/19 (32\%) cell lines chondrogenic differentiation was evident (Figure 1).

\section{Cell Line-Derived Tumors Represent Osteosarcoma}

Eight out of 19 osteosarcoma cell lines tested produced tumors in vivo after subcutaneous and intramuscular injections (Figure $2 \mathrm{a}-\mathrm{h}$ ), intramuscular tumors formed on average twice as fast compared with the tumors produced by the same cells injected subcutaneously. All tumors were characterized as high-grade sarcomas and, although not always abundant, tumor cells produced osteoid that is characteristic of osteosarcoma. The tumor produced by one of the oldest human-derived cell lines, U2OS, showed abundant osteoid production (Figure 2i) and infiltrating immune cells were detected (Figure 2n). The IOR/OS9 cells showed differentiation in vivo (Figure 2e, $\mathrm{j}$ and o). Furthermore, six cell lines were shown to invade into adjacent tissues and in all recipient animals angiogenesis was found. The HOS-143B cell line was tumorigenic and metastatic (Figure 3). 
Table 2 In vitro functional analysis and in vivo tumor progression

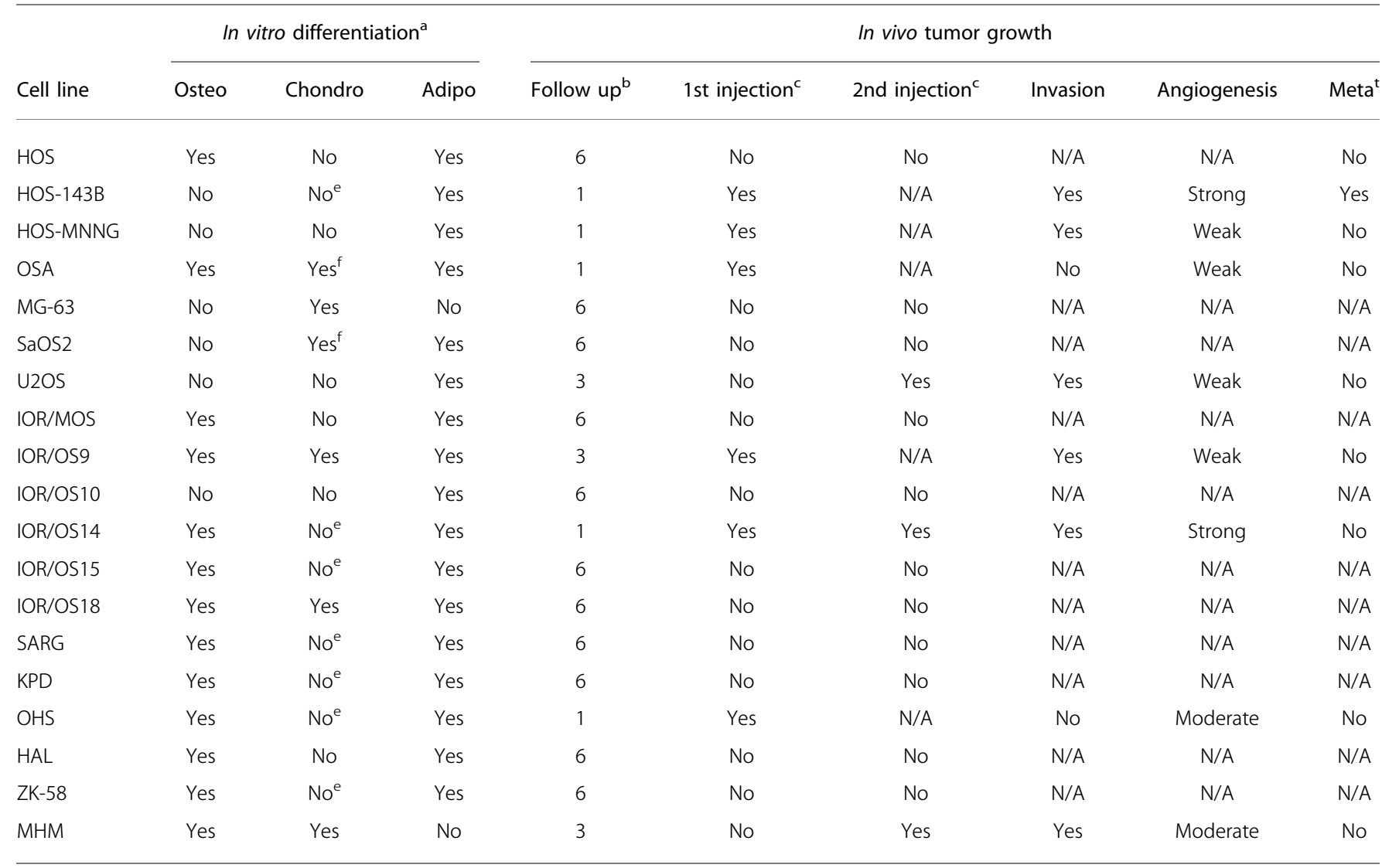

N/A, Not available.

This table summarizes whether cells could differentiate in vitro and specifies the lineage(s) of differentiation. On the right part is indicated whether a cell line was tumorigenic in vivo under the described circumstances, and if Yes, the occurrence and extent of invasion to adjacent tissues and angiogenesis are indicated. Please Note that injected animals were observed for 6 months if tumors were Not found before.

ahether cells could be pushed towards osteogenic, chondrogenic and/or adipogenic differentiation.

${ }^{b}$ Time in months from injection to killing of the mice.

${ }^{c}$ Whether tumor formation was seen after a first or second round of injection of the cells.

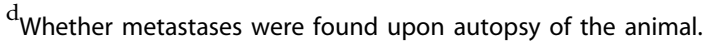

${ }^{\mathrm{e}}$ Cells could Not form a pellet.

fWeakly positive.

\section{Protein Expression Profiles by Immunohistochemistry}

Because of the unavailability of specific markers for osteosarcoma, tissue arrays from primary xenografts and metastases were stained for a panel of proteins described to be associated with the tumor in different processes (Table 3):

- differential differentiation: osteonectin $(\mathrm{ON})$, osteocalcin (BGLAP), vimentin (VIM), cytokeratin (CK), epithelial membrane antigen (EMA), CD34, smooth muscle actin (SMA), desmin (DES) and transcription factor SOX9 (SOX9);

- oncogenesis: CD99, B-cell lymphoma protein 2 (BCL2), C-Kit (KIT), caveolin 1 (CAV1), Ki-67, TP53 (p53),
CDKN2A (p16), CDKN1A (p21), epidermal growth factor receptor (EGFR), HER2, tumor protein D52 (TPD52) and S100; and

- invasion/migration: ezrin (EZR), e-cadherin (E-Cad) and CD31.

The immunohistochemical expression profiles of xenografts from different cell lines show similar patterns of protein expression reminiscent of primary human osteosarcoma as known from the literature. This pattern is maintained in the metastases as well, indicating a homogeneous cell population. The expression of the proteins described here did not correlate with differentiation capacity or tumorigenicity of the cell lines. 


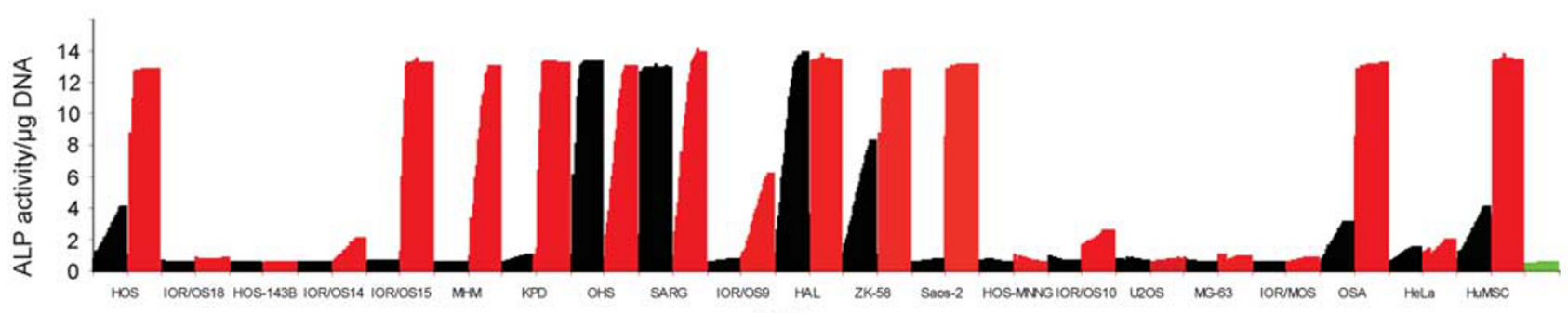

Cell lines
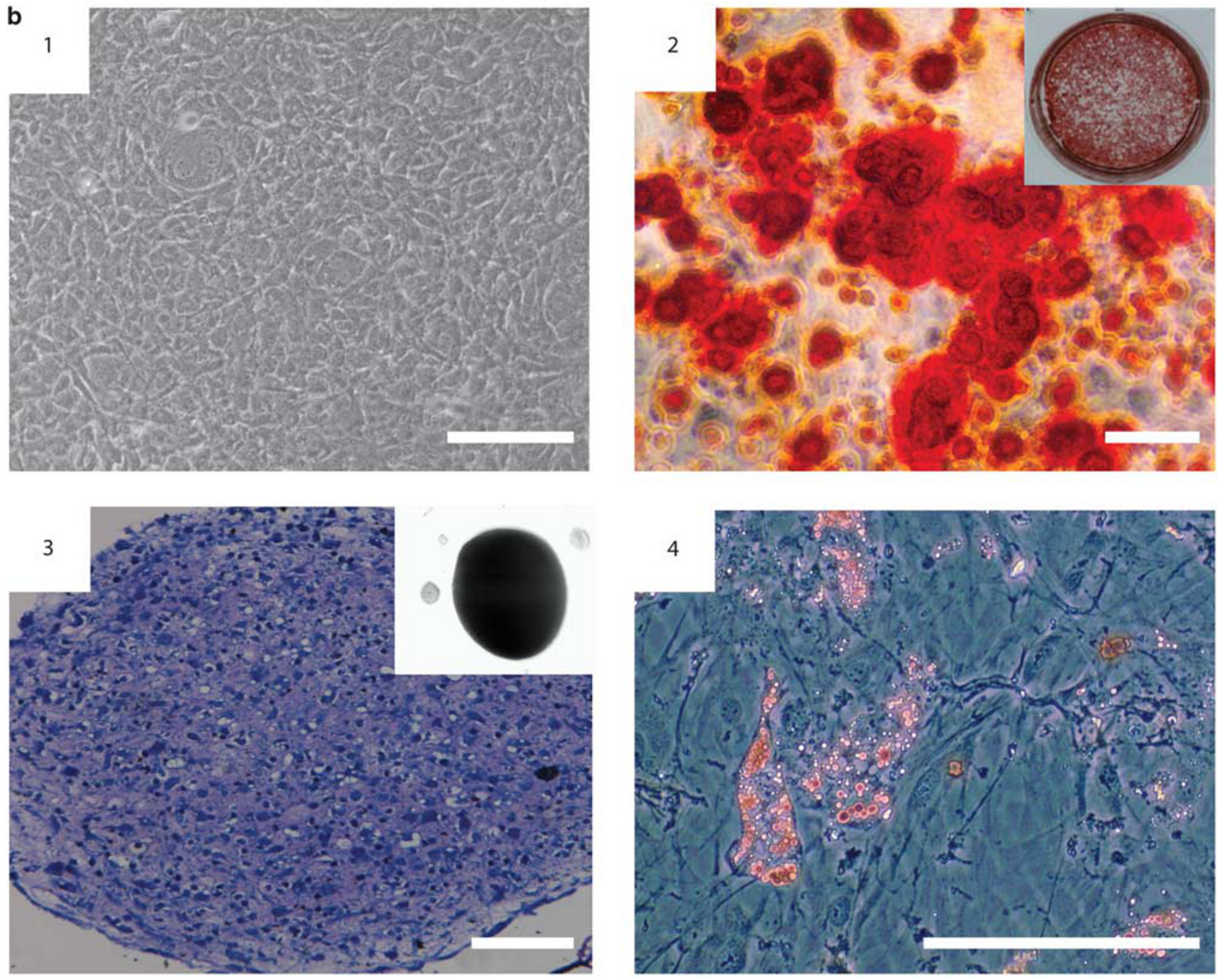

Figure 1 Functional characterization of the cells by multi-lineage differentiation assays. (a) The quantitative alkaline phosphatase (ALP) activity per $\mu \mathrm{g}$ DNA is depicted. For each cell line, the activity was measured before, to assay basal activity, and after 7 days of differentiation. Enzyme activity was recorded during $3 \mathrm{~h}$, in general showing increase at the initial phase after which saturation and stable activity followed. HeLa and a primary human mesenchymal stem cell culture (HuMSC) were used as positive and negative controls, respectively. On the basis of the ALP activity, cell lines can be divided into three groups: (1) cells that do not show basal activity before differentiation and which does not increase after 7 days of differentiation; (2) cells that do not show basal activity, but this does increase significantly after differentiation and (3) cells that show high ALP activity before and after differentiation. (b) Representative pictures of cell cultures showing the morphology of IOR/OS18 cells at confluence before differentiation (1), mineral staining of HOS cells by Alizarin Red S (2), a paraffin section of MG-63 pellets induced to differentiate towards chondrocytes and stained by toluidine blue (3) and adipocytes, differentiated from HOS-MNNG cells, containing lipid droplets stained by Oil Red O (4). Bars represent $100 \mu \mathrm{m}$, and in the insets, the wells from the same samples are depicted. 

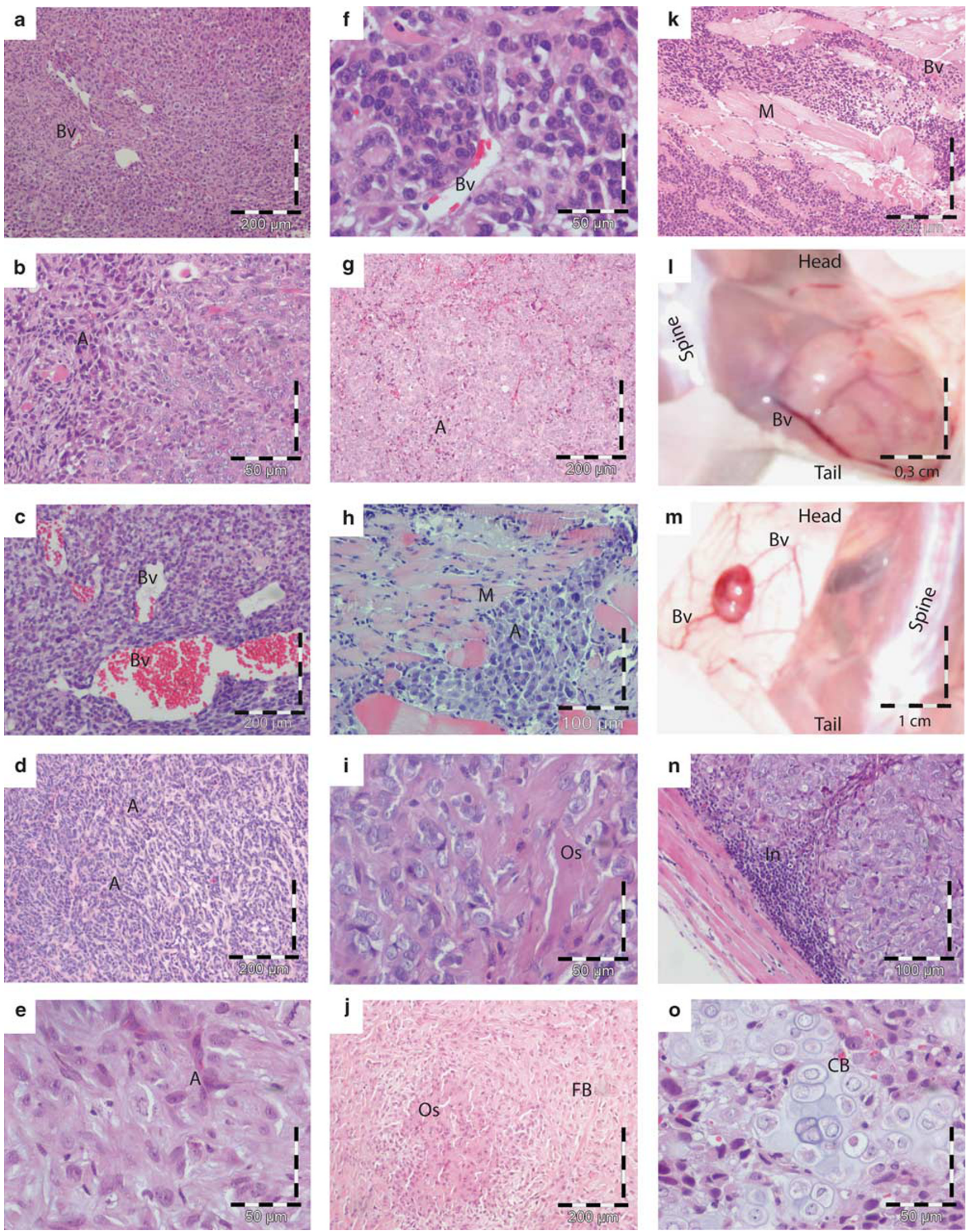


\section{HOS-143B: A Metastasizing Cell Line}

After autopsy of the mouse injected subcutaneously and intramuscularly with HOS-143B cells, multiple lung metastases were detected. Subsequently, five mice were injected only subcutaneously (at two locations making a total of 10 injections) and an additional 10 mice only intramuscularly, of which, respectively, 3 and 10 again rapidly (3-6 weeks after detection of the primary tumor) developed lung metastases. Moreover in one animal, which received an intramuscular injection of $2 \times 10^{6}$ HOS-143B cells, brain metastases were detected (Figure 3). Detailed examination of the mice and the tumors confirmed that the metastases were not caused by accidentally intravenous injections. Furthermore, a piece of one of the tumors was xenografted into another nude mouse; this process was repeated three times, that is, passaged three times in vivo, after which the tumor was still metastatic. The xenografts and the metastases were shown to have high expression of the p53 protein in line with the previously detected p53 mutation in the originating HOS-143B cell line. ${ }^{18}$ Here p53 mutation analysis showed that this p.Arg156Pro mutation was also present in the metastases.

\section{DISCUSSION}

Osteosarcoma is a malignant disease of the childhood and improvements in the survival rates have reached a plateau phase. This is partly due to a lack of good understanding of the biology of this complex tumor given its rareness and the high genetic heterogeneity and instability at diagnosis. To overcome this problem, the establishment of novel osteosarcoma models and a good characterization of the existing models is essential. Tumor-derived cell lines could be used as excellent in vitro and in vivo models as long as they are representative of the original tumor. For this we assayed here all, genetically characterized, osteosarcoma cell lines to identify the ones most representative for clinical osteosarcoma that could be used to establish valid in vitro and in vivo models.

Although it has been reported that cell lines represent most of their parental tumors characteristics, they are questioned because of the additional (in vitro obtained) genetic alterations ${ }^{8,14}$ and to what extent the cell lines are equal to the original tumors. Here, we show that a number of osteosarcoma cell lines can produce growing and in one case even metastasizing tumors under technically feasible circum- stances. We hypothesize that after years of cultivation, the cultures become more homogeneous based on clonal selection, slowly resulting into more stabilized genetics, and in some cases resuming in the loss of tumorigenic potential. The main aim of this study was to identify cell lines that are both known to be genetically stable providing useable models and still able of producing tumors representative of human osteosarcoma.

Eight cell lines were identified, which gave rise to tumors after injection into immune-deficient mice. Given their histology and osteoid production, the tumors were shown to be representative of osteosarcoma. Furthermore, a spectrum of different important features of tumorigenesis was assessed in the xenografts, identifying proper cell lines to model immune attraction (U2OS), angiogenesis (IOR/OS-14 and HOS$143 \mathrm{~B})$, invasion to adjacent tissues (MHM), in vivo differentiation (IOR/OS9) and metastasis (HOS-143B). Here we focused on HOS-143B as metastatic osteosarcoma models are exceptional, ${ }^{36}$ and a metastatic human-derived osteosarcoma cell line could model the metastasizing process of osteosarcoma, which is the main clinical issue for the patient. This cell line was already reported to be metastatic in an orthotopic model $;{ }^{37}$ however, its rapid metastasizing potential in a technically more manageable setting, that is, subcutaneous injection as shown here, is new. Moreover, even after transplanting a piece of the primary xenograft into a new recipient and repeating this up to three times, still metastases rapidly could be detected in the lungs of the animals. This indicates metastatic potential and excludes that the lung metastases are a result of migrating cells after injection of a loose cell mass into the mice. The availability of HOS-143B non-tumorigenic parental cell line HOS and its tumorigenic, but nonmetastatic progeny cell line HOS-MNNG, ${ }^{38}$ makes these three cell lines excellent models to comparatively study osteosarcoma progression and metastasis, respectively. For example, the TP53 mutation found in all three cell lines might suggest that $\mathrm{p} 53$ has a role in tumor initiation, but not that much in progression and metastasis, and that additional events (HOS-MNNG was generated by the chemical agent $\mathrm{N}$ methyl- $N^{\prime}$-nitro- $N$-nitroguanidineare and HOS-134B via a Ki-ras oncogene transformation) are needed. This might explain the lack of association between TP53 mutation and metastasis or outcome in osteosarcoma patients. ${ }^{39,40}$

Figure 2 In vivo characterization of the cell lines. Hematoxylin and eosin (H\&E)-stained sections of the tumors resulting from subcutaneous and intramuscular injections of cell lines into nude mice are shown. (a-h) Primary xenografts of the eight tumorigenic cell lines: HOS-143B (a), HOS-MNNG (b), OSA (c), U2OS (d), IOR/OS9 (e), IOR/OS14 (f), OHS (g) and MHM (h) are represented, respectively. (i) A section of the tumor produced by U2OS cells (one of the oldest human-derived cell lines), which shows abundant osteoid (Os) production. (j, o) Two sections from a single IOR/OS9 originating tumor, which are next to the osteoblastic areas (e) illustrate fibroblastic and chondroblastic differentiation, respectively, showing in vivo differentiation of the cells. (k) After subcutaneous injection of and tumor formation by IOR/OS14 cells, this picture shows aggressive invasion of the tumor cells into the adjacent tissues. (I, $\mathbf{m}$ ) Macroscopical pictures of intramuscular (HOS-MNNG) and subcutaneous (OHS) tumors, respectively. Please note the formation of big blood vessels towards the tumors indicating angiogenesis. In ( $\mathbf{m})$, the skin of the mouse is cut and flipped open to the side, which shows that the tumor is isolated in the subcutane and that injection did not accidentally penetrate the underlying tissues. (n) Picture is showing a part of the U2OS tumor where repeatedly infiltrating native immune cells were identified. Abbreviations: osteoid (Os), atypical tumor cells (a), blood vessels indicating neo-vascularization (Bv), muscle (M), infiltrating cells (In), fibroblastic matrix (FB) and chondroblastic matrix (CB). 
a
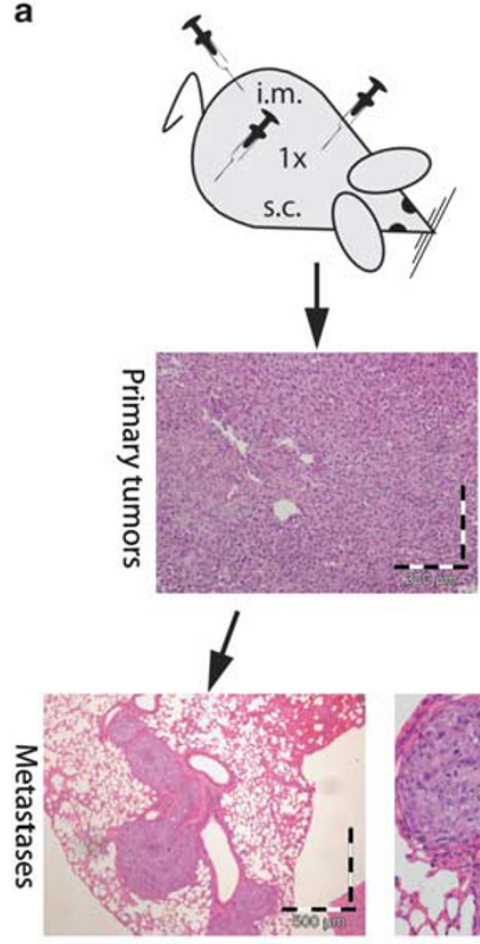

$x$
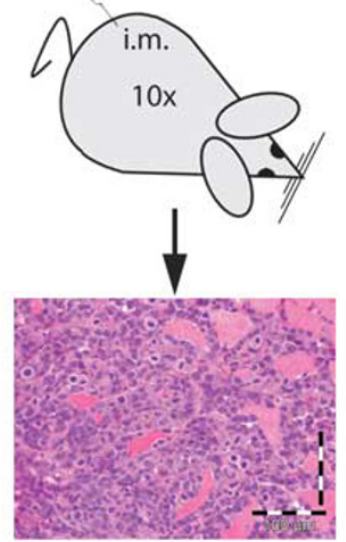
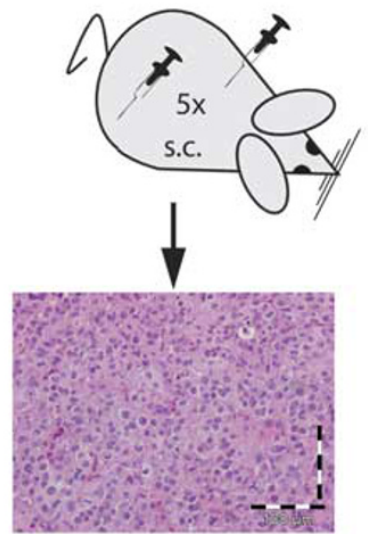

C
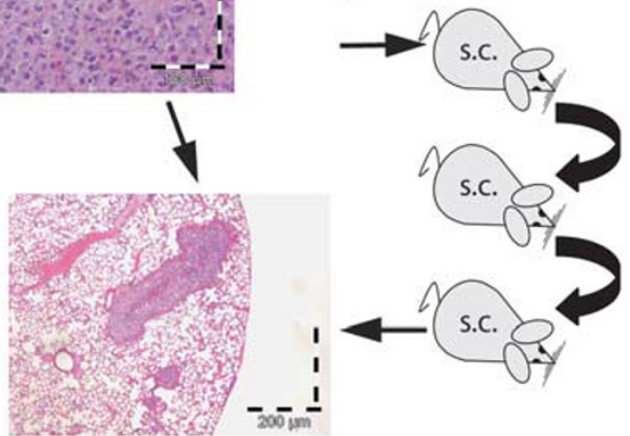

b

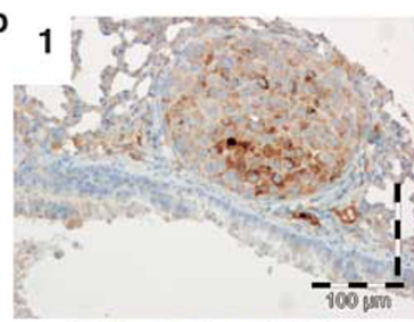

2 is

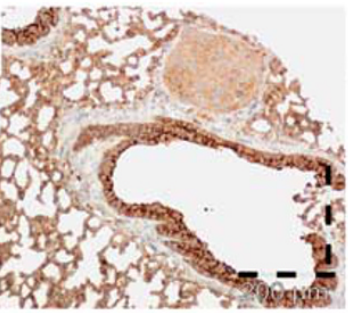

3
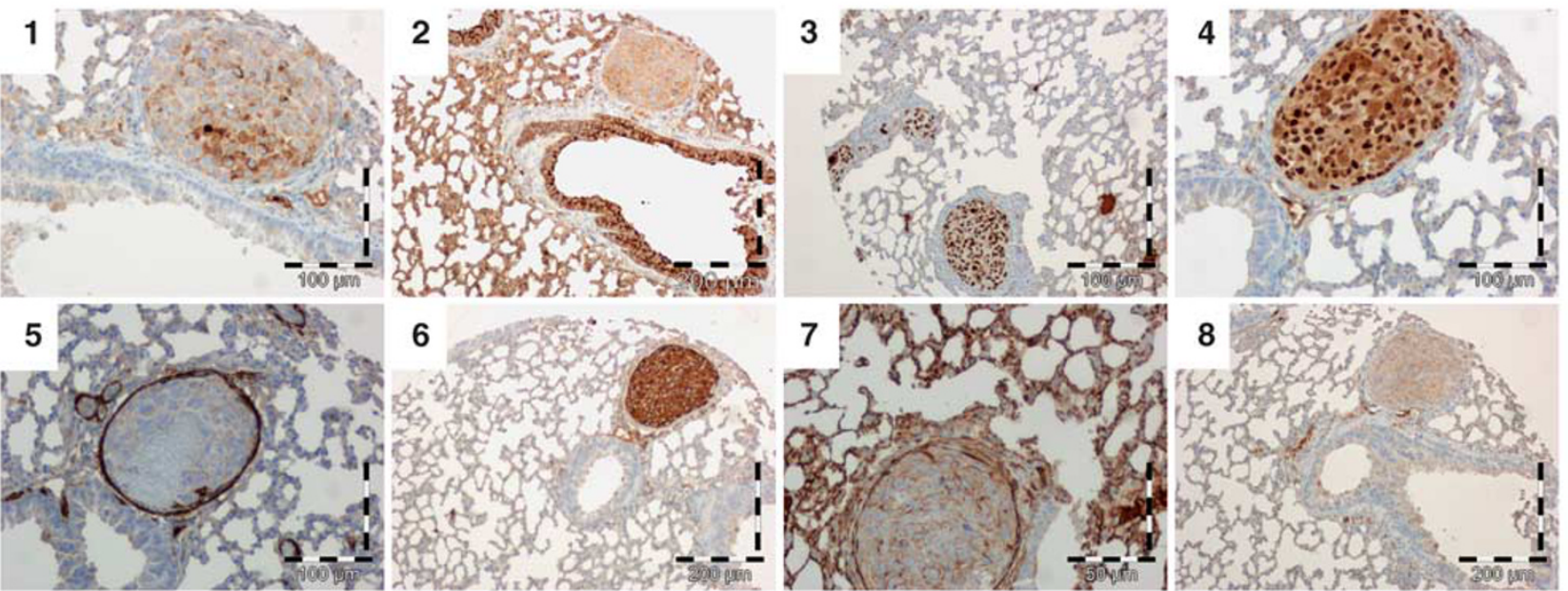

\section{6}

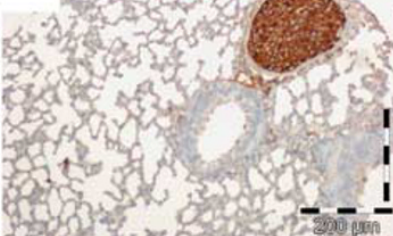

\section{7}
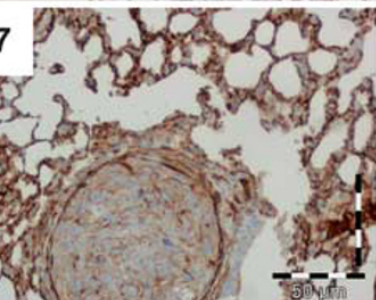

8
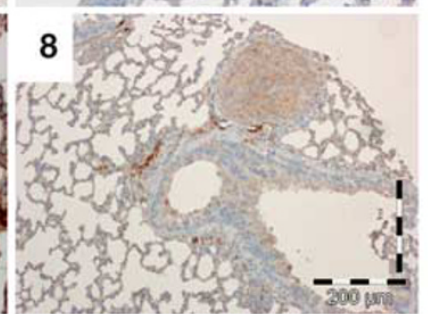

Figure 3 Metastatic HOS-143B cell line. (a) After subcutaneous (s.c.) and intramuscular (i.m.) injection of HOS-143B cells into one nude mouse, lung metastases were detected. To determine whether i.m. or s.c. tumors or both were metastatic, respectively, 10 and 5 animals were solely i.m. and solely s.m. injected. The primary tumors after these injections in the animals again led to the formation of multiple lung metastases and in one case a brain metastasis was found as indicated by ${ }^{*}$. (b) Sections 1-8 show representative images of the immunohistochemical staining of HOS-143B lung metastases. Pictures, respectively, depict: osteonectin, ezrin, Ki-67, p53, smooth muscle actin, vimentin, caveolin 1 and CD99. (c) To confirm that the HOS-143B-derived tumors are actively metastasizing after the primary tumor is formed and that lung and brain metastases are not only a result of migrating cells just after injection, a small piece of one of the tumors was subcutaneously transplanted into a new recipient mouse. After the tumor again grew up to $1 \mathrm{~cm}$, the procedure was repeated. Even after three subsequent repetitions, metastases were still detected in the lungs of the animal.

The system used here, subcutaneous and intramuscular injections of human osteosarcoma cell lines into immunedeficient mice, did not result in tumor formation in 11 out of 19 cell lines. A second attempt of injecting these 11 cell lines in new recipient mice confirmed their inability of generating tumors under these circumstances. This might indicate that these cell lines have lost their tumorigenicity because of long- term selection for other characteristics important to survive the in vitro culturing conditions or that the lines are originating from other cell populations in the initial heterogeneous cell culture than the osteosarcoma cells. Alternatively, this might reflect the dissimilar microenvironment used here (under the skin and inside the muscle) compared with the intramedullar locations where mostly 
Table 3 Immunohistochemical analysis of primary the xenografts and metastases

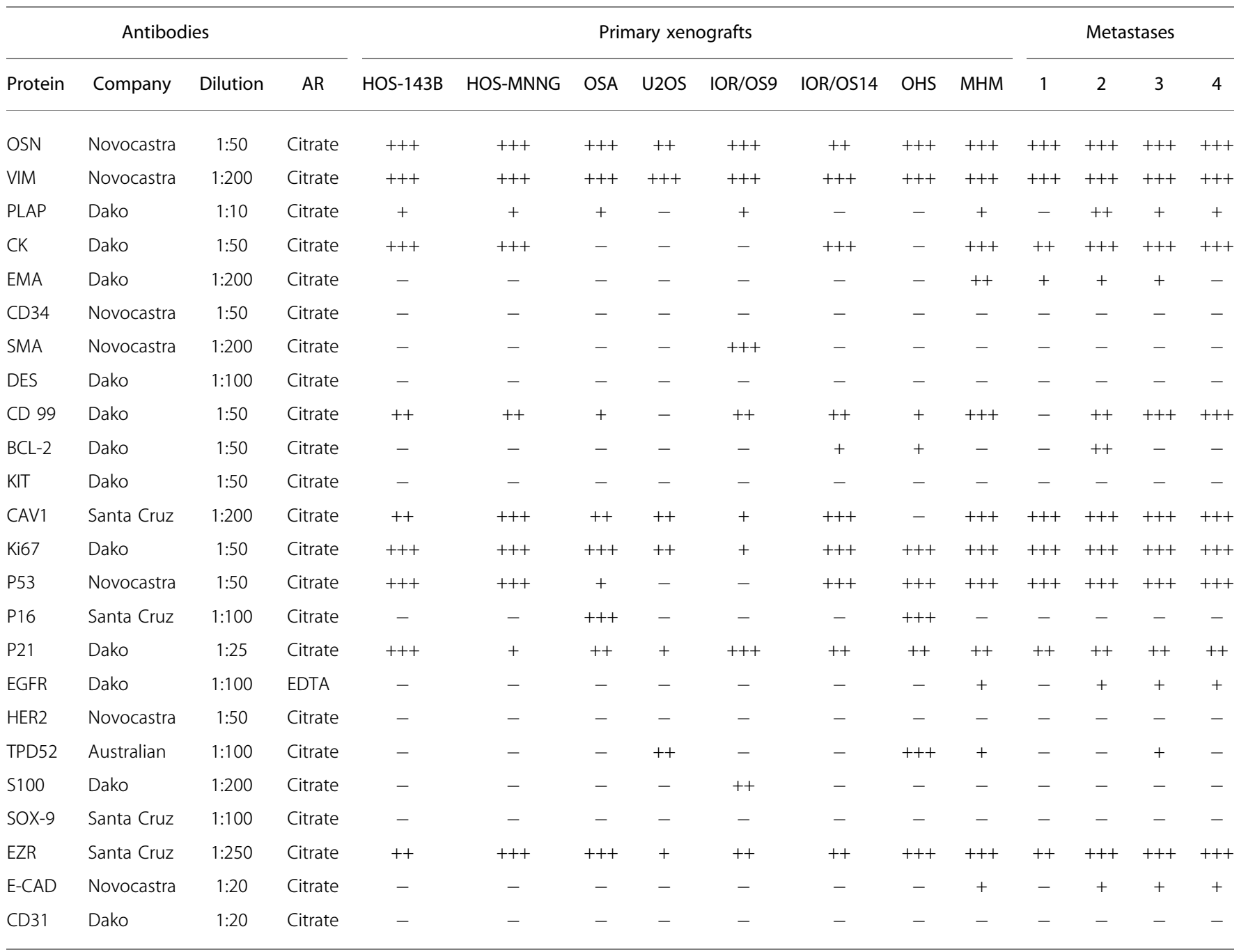

- , negative; + , weakly positive; ++ , moderate positive; +++ , strongly positive.

The first four columns are depicting the antibodies' characteristics and the immunohistochemical staining conditions. Furthermore, the staining patterns of the xenografts and four HOS-143B metastases are shown.

osteosarcoma is found, indicating that we cannot exclude the tumorigenicity of these cell lines by other techniques as orthotopic injections. Therefore, this study was especially useful for selecting cell lines that are tumorigenic under simplified conditions to identify technically practical models. Moreover, the limited in vivo lineage-specific differentiation of the cell lines underlines the importance of (stromal or microenvironmental) stimulation for this process as most cell lines do differentiate in vitro.

Recently, the use of cell lines has been questioned again as reports show high incidence of cross-contaminations between cell lines with all disastrous consequences, ${ }^{41}$ for which the ATCC has developed a cell line identification standard. ${ }^{42}$ One advantage of the cell lines series used here is that they all were previously characterized at genetic level, ${ }^{18}$ thereby allowing for checking cell identities and excluding cross-contaminations. In this study, all cell lines were genotyped at the end of the experiments to match with their initial profiles and all xenografts were genotyped to match with the profiles of the originating cell lines. From our extensive experience, especially with these highly proliferative cells, and the ongoing debate in literature, we strongly recommend regular-based DNA profiling of cell lines. Any cell line, as long as it is in culture, should regularly undergo quality checks to confirm its identity and pathogen-free state, as cross-contamination with more aggressive cell lines, like HeLa and HOS, and animal cell lines can happen even in best hands.

For the past 30 years, study of osteosarcoma has widened our knowledge about this aggressive malignancy. The high genetic instability of the primary tumor, the rareness of the 
disease and poor access to primary patient material due to intensive treatment regimens hamper biological studies. Therefore, multiple representative models are needed to get more insight into different processes involving osteosarcoma initiation, progression and treatment. Next to novel models that could shed light to osteosarcoma initiation, we hypothesized that after extensive biological and genetical characterization, osteosarcoma cell lines could provide good models to study osteosarcoma progression and treatment. We were able to show (multi-lineage) differentiation capacity of nearly all osteosarcoma cell lines. This might indicate the stemness of these tumors providing more knowledge about their origin and its useful information to investigate the role of differentiation in tumorigenesis. After identifying tumorigenic cell lines under simplified conditions, we could pinpoint a number of cell lines that could be used as models for specific research questions.

\section{ACKNOWLEDGEMENTS}

We thank Elisa Alonso, Silvia Calabuig-Fariñas, Laura Lopez, Marcel Winter, Diane Kagabo, Brendy van den Akker, Maayke van Ruler, Pauline WijersKoster and Inge Briaire-de Bruijn for technical assistance. Furthermore, we thank Drs Ola Myklebost (Radiumhospitalet, Oslo) for providing the MHM cell line and expression data, Jeniffer Byrne (The Children's Hospital, Sydney) for providing the TPD52 antibody and Marieke Kuijjer (Leiden University Medical Center, Leiden) for sharing and analyzing unpublished expression data. This work was supported by EuroBoNet, a European Commission granted Network of Excellence for studying the pathology and genetics of bone tumors (Grant LSHC-CT-2006-018814).

\section{DISCLOSURE/CONFLICT OF INTEREST}

The authors declare no conflict of interest.

1. Masters JR. HeLa cells 50 years on: the good, the bad and the ugly. Nat Rev Cancer 2002;24:315-319.

2. Chu D, Zheng J, Wang W, et al. Notch2 expression is decreased in colorectal cancer and related to tumor differentiation status. Ann Surg Oncol 2009.

3. Zhong Y, Onuki J, Yamasaki T, et al. Genome-wide analysis identifies a tumor suppressor role for aminoacylase 1 in iron-induced rat renal cell carcinoma. Carcinogenesis 2009;301:158-164.

4. $\mathrm{Xu}$ L, Ding $\mathrm{Y}$, Catalona WJ, et al. MEK4 function, genistein treatment, and invasion of human prostate cancer cells. J Nat Cancer Inst 2009.

5. Xu Z, Chen ZP, Malapetsa A, et al. DNA repair protein levels vis-a-vis anticancer drug resistance in the human tumor cell lines of the National Cancer Institute drug screening program. Anticancer Drugs 2002;135:511-519.

6. Scheper MA, Shirtliff ME, Meiller TF, et al. Farnesol, a fungal quorumsensing molecule triggers apoptosis in human oral squamous carcinoma cells. Neoplasia 2008;109:954-963.

7. Vescio RA, Connors KM, Kubota T, et al. Correlation of histology and drug response of human tumors grown in native-state threedimensional histoculture and in nude mice. Proc Natl Acad Sci USA 1991;8812:5163-5166.

8. Sharma SV, Haber DA, Settleman J. Cell line-based platforms to evaluate the therapeutic efficacy of candidate anticancer agents. Nat Rev Cancer 2010;104:241-253.

9. Arlett CF. The use of dubious cell lines in research: is trust enough? Lancet Oncol 2001;28:467.

10. Drexler HG, Dirks WG, Matsuo Y, et al. False leukemia-lymphoma cell lines: an update on over 500 cell lines. Leukemia 2003;172:416-426.

11. Masters JR. Human cancer cell lines: fact and fantasy. Nat Rev Mol Cell Biol 2000;13:233-236.
12. Drexler HG, Uphoff CC, Dirks WG, et al. Mix-ups and mycoplasma: the enemies within. Leuk Res 2002;264:329-333.

13. Webster JA, Beck AH, Sharma $M$, et al. Variations in stromal signatures in breast and colorectal cancer metastases. J Pathol 2010;222:158-165.

14. Greshock J, Nathanson K, Martin AM, et al. Cancer cell lines as genetic models of their parent histology: analyses based on array comparative genomic hybridization. Cancer Res 2007;678:3594-3600.

15. Ross DT, Scherf $U$, Eisen $M B$, et al. Systematic variation in gene expression patterns in human cancer cell lines. Nat Genet 2000;243:227-235.

16. Tinker AV, Boussioutas A, Bowtell DD. The challenges of gene expression microarrays for the study of human cancer. Cancer Cell 2006;95:333-339.

17. Douglas EJ, Fiegler $\mathrm{H}$, Rowan $\mathrm{A}$, et al. Array comparative genomic hybridization analysis of colorectal cancer cell lines and primary carcinomas. Cancer Res 2004;6414:4817-4825.

18. Ottaviano L, Schaefer KL, Gajewski M, et al. Molecular characterization of commonly used cell lines for bone tumor research: a trans-European EuroBoNet effort. Genes Chromosomes Cancer 2010;491:40-51.

19. Stratton MR, Campbell PJ, Futreal PA. The cancer genome. Nature 2009;4587239:719-724.

20. Sandberg R, Ernberg I. Assessment of tumor characteristic gene expression in cell lines using a tissue similarity index (TSI). Proc Natl Acad Sci USA 2005;1026:2052-2057.

21. Benini S, Baldini N, Manara MC, et al. Redundancy of autocrine loops in human osteosarcoma cells. Int J Cancer 1999;804:581-588.

22. Bruland $\mathrm{OS}$, Fodstad $\mathrm{O}$, Stenwig $\mathrm{AE}$, et al. Expression and characteristics of a novel human osteosarcoma-associated cell surface antigen. Cancer Res 1988;4818:5302-5309.

23. Fodstad O, Brogger A, Bruland O, et al. Characteristics of a cell line established from a patient with multiple osteosarcoma, appearing 13 years after treatment for bilateral retinoblastoma. Int J Cancer 1986;381:33-40.

24. Schulz A, Battmann A, Heinrichs CM, et al. Properties and reactivity of a new human osteosarcoma cell line (HOS 58). Calcif Tissue Int 1993;52:30.

25. Kjonniksen I, Winderen $\mathrm{M}$, Bruland $\mathrm{O}$, et al. Validity and usefulness of human tumor models established by intratibial cell inoculation in nude rats. Cancer Res 1994;547:1715-1719.

26. Lombaerts $\mathrm{M}$, van WT, Philippo K, et al. E-cadherin transcriptional downregulation by promoter methylation but not mutation is related to epithelial-to-mesenchymal transition in breast cancer cell lines. $\mathrm{Br} J$ Cancer 2006;945:661-671.

27. Mohseny $A B$, Szuhai $K$, Romeo $S$, et al. Osteosarcoma originates from mesenchymal stem cells in consequence of aneuploidization and genomic loss of Cdkn2. J Pathol 2009;2193:294-305.

28. van der Horst G, van Bezooijen RL, Deckers MM, et al. Differentiation of murine preosteoblastic KS483 cells depends on autocrine bone morphogenetic protein signaling during all phases of osteoblast formation. Bone 2002;316:661-669.

29. Kononen J, Bubendorf L, Kallioniemi $A$, et al. Tissue microarrays for high-throughput molecular profiling of tumor specimens. Nat Med 1998;47:844-847.

30. Goethals L, Perneel C, Debucquoy A, et al. A new approach to the validation of tissue microarrays. J Pathol 2006;2085:607-614.

31. Bovee JVMG, Van den Broek LCM, Cleton-Jansen AM, et al. Upregulation of PTHrP and $\mathrm{BCl}-2$ expression characterizes the progression of osteochondroma towards peripheral chondrosarcoma and is a late event in central chondrosarcoma. Lab Invest 2000;80:1925-1933.

32. Romeo S, Bovée JVMG, Jadnanansing NAA, et al. Expression of cartilage growth plate signalling molecules in chondroblastoma. J Pathol 2004:2021:113-120.

33. Detre S, Saccani Jotti G, Dowsett M. A 'quickscore' method for immunohistochemical semiquantitation: validation for oestrogen receptor in breast carcinomas. J Clin Pathol 1995;489:876-878.

34. Da Costa $C E$, Szuhai $K$, van Eijk $R$, et al. No genomic aberrations in Langerhans cell histiocytosis as assessed by diverse molecular technologies. Genes Chromosomes Cancer 2009:483:239-249.

35. van Eijk $R$, van Puijenbroek $M$, Chhatta $A R$, et al. Sensitive and specific KRAS somatic mutation analysis on whole-genome amplified DNA from archival tissues. J Mol Diagn 2010;12:27-34. 
36. Ek ET, Dass CR, Choong PF. Commonly used mouse models of osteosarcoma. Crit Rev Oncol Hematol 2006;601:1-8.

37. Luu $\mathrm{HH}$, Kang $\mathrm{Q}$, Park JK, et al. An orthotopic model of human osteosarcoma growth and spontaneous pulmonary metastasis. Clin Exp Metast 2005;224:319-329.

38. Dass CR, Ek ET, Choong PF. Human xenograft osteosarcoma models with spontaneous metastasis in mice: clinical relevance and applicability for drug testing. J Cancer Res Clin Oncol 2007;1333:193-198.

39. Wunder JS, Gokgoz N, Parkes R, et al. TP53 mutations and outcome in osteosarcoma: a prospective, multicenter study. J Clin Oncol 2005;237: 1483-1490.
40. Gokgoz N, Wunder JS, Mousses S, et al. Comparison of p53 mutations in patients with localized osteosarcoma and metastatic osteosarcoma. Cancer 2001;928:2181-2189.

41. Torsvik A, Rosland GV, Svendsen A, et al. Spontaneous malignant transformation of human mesenchymal stem cells reflects crosscontamination: putting the research field on track-letter. Cancer Res 2010;7015:6393-6396.

42. American Type Culture Collection Standards Development Organization Workgroup ASN-0002Alston-Roberts C Barallon R, Bauer $\mathrm{SR}$, et al. Cell line misidentification: the beginning of the end. Nat Rev Cancer 2010;106:441-448. 